\title{
PEAK POINTS, BARRIERS AND PSEUDOCONVEX BOUNDARY POINTS ${ }^{1}$
}

\author{
RICHARD F. BASENER
}

\begin{abstract}
Let $x$ be a smooth boundary point of a domain in $\mathbf{C}^{n}$. It is shown that $x$ is a limit of strictly pseudoconvex boundary points whenever there is a "plurisubharmonic barrier" for $x$.
\end{abstract}

1. Introduction. Let $D$ be an open subset of $\mathbf{C}^{n}$ and, as usual, let

$$
A(D)=\{f \in C(\bar{D}) \mid f \text { is holomorphic on } D\} \text {. }
$$

If $D$ is bounded then $A(D)$ is a uniform algebra on $\bar{D}$ and in this case we let $S(D)$ denote the Shilov boundary of $A(D)$, a subset of $\partial D$.

The problem of characterizing $S(D)$ for certain pseudoconvex domains was discussed by Bremermann in [1, Theorems 6.8 and 6.9]. Related results were obtained by Rossi in [11]. Most recently Pflug has used the results of Kohn in [6] to show that $S(D)$ contains the closure of the set of strictly pseudoconvex boundary points of $D$ when $D$ is a psuedoconvex domain with $C^{\infty}$ boundary (see Folgerung 5 in [10]); Pflug refers to [3] for the reverse inclusion, but this reference does not seem to be widely available. Debiard and Gaveau have shown that $S(D)$ is contained in the closure of the strictly pseudoconvex boundary points when $D$ has the form $\{z \mid V(z)<0\}$ for some $C^{3}$ function $V$ defined near $\bar{D}$ which is plurisubharmonic on $D$ and satisfies $d V \neq 0$ on $\partial D$. They follow earlier work of Malliavin [7] [9] and E. M. Stein [12] in applying probabilistic potential theory to study boundary behavior in $\mathbf{C}^{n}$ via suitable Kählerian metrics (see [2]).

In this note we prove that $S(D)$ is contained in the closure of the strictly pseudoconvex boundary points of $D$ when $D$ is any bounded open subset of $\mathbf{C}^{n}$ with $C^{2}$ boundary. The same kind of elementary geometric considerations which Rossi utilized in [11] can be used to obtain this sharper result. (Much the same result is part of a recent announcement of Hakim and Sibony [4].) We actually show that a boundary point which has a "plurisubharmonic barrier" is a limit of strictly pseudoconvex boundary points (Corollary 1), from which the partial characterization of $S(D)$ follows at once.

2. Preliminaries. Let $D$ be an open subset of $\mathbf{C}^{n}$. A defining function for $D$ on a set $\Omega \subseteq \mathbf{C}^{n}$ is a real-valued function $\phi$ on $\Omega$ such that $D \cap \Omega=\{\phi<0\}$.

Received by the editors March 12, 1976.

AMS (MOS) subject classifications (1970). Primary 32F15.

'Research supported in part by NSF Grant MPS75-07922.

O American Mathematical Society 1977 
If $\alpha \in \partial D, \alpha$ is a $C^{2}$ boundary point of $D$ if there is a neighborhood $\Omega$ of $\alpha$ and a $C^{2}$ defining function $\phi$ for $D$ on $\Omega$ with $(d \phi)_{\alpha} \neq 0$. Given such an $\alpha$, we may choose coordinates $z_{j}=x_{j}+i y_{j}$ for $\mathbf{C}^{n}$ so that $\alpha=0$ and $(d \phi)_{\alpha}=$ $\left(d x_{1}\right)_{\alpha}$, whence the tangent space to $\partial D$ at $\alpha$ is $\left\{x_{1}=0\right\}$. If we make the identification $\mathbf{C}^{n}=\mathbf{R} \times\left(\mathbf{R} \times \mathbf{C}^{n-1}\right)$ by $z=\left(x_{1},\left(y_{1},\left(z_{2}, \ldots, z_{n}\right)\right)\right)$, then the implicit function theorem enables us to choose a $\delta>0$, an open neighborhood $U$ of 0 in $\mathbf{R} \times \mathbf{C}^{n-1}$, and a real-valued function $\psi \in C^{2}(U)$ with the property that

$$
D \cap[(-\delta, \delta) \times U]=\{(x, t) \in(-\delta, \delta) \times U \mid x<\psi(t)\}
$$

Since $x-\psi(t)$ is a defining function for $D$ on $(-\delta, \delta) \times U$ whose restriction to the complex tangent space to $\partial D$ at $\alpha$ is $-\psi\left(0,\left(z_{2}, \ldots, z_{n}\right)\right)$, $\alpha$ will be a strictly pseudoconvex boundary point of $D$ precisely if $\psi$ is strictly superharmonic at 0 on every complex line through 0 in $U$.

Suppose $K$ is a compact smoothly bounded subset of $\mathbf{R}^{n}, B$ is a closed ball, $B \supset K$, and $\alpha \in \partial B \cap \partial K$; then of course $\alpha$ is a strictly convex boundary point of $K$. The following proposition (proved by Rossi in [11]) is an intuitively obvious complex analogue of this result, and provides us with a criterion for recognizing strictly pseudoconvex boundary points.

Proposition. Let $D$ be an open subset of $\mathbf{C}^{n}, \alpha$ a $C^{2}$ boundary point of $D, \Omega$ an open neighborhood of $\alpha, v \in C_{\mathbf{R}}^{2}(\Omega)$, $v$ strictly plurisubharmonic. Suppose that $v(\alpha)=0$ and that $v<0$ on $D \cap \Omega$. Then $\alpha$ is a strictly pseudoconvex boundary point of $D$.

Proof. We may assume that $(d v)_{\alpha} \neq 0$; for if $(d v)_{\alpha}=0$ then one can replace $v$ by $v+\varepsilon \phi$, where $\phi$ is any defining function for $D$ near $\alpha$ with $(d \phi)_{\alpha} \neq 0$ and $\varepsilon$ is a corresponding small positive number. Choose coordinates for $\mathbf{C}^{n}$ so that $\alpha=0$ and $(d v)_{\alpha}=\left(d x_{1}\right)_{\alpha}$. Then there is an open set $V$ in $\mathbf{C}^{n}$ with $\alpha \in V \subseteq \Omega$, and an open neighborhood $U$ of 0 in $\mathbf{R} \times \mathbf{C}^{n-1}$ with functions $\psi, u \in C_{\mathbf{R}}^{2}(U)$, such that

$$
\begin{gathered}
D \cap V=\{(x, t) \in \mathbf{R} \times U \mid x<\psi(t)\} ; \\
\{v<0\} \cap V=\{(x, t) \in \mathbf{R} \times U \mid x<u(t)\} ; \\
\{(\psi(t), t) \mid t \in U\} \subseteq V .
\end{gathered}
$$

Note that $\psi(0)=u(0)=0$ since $\alpha \in \partial D \cap \partial(\{v<0\})$. Furthermore, $\psi \leqslant u$. [Let $t \in U$. Then for any small positive $\varepsilon,(\psi(t)-\varepsilon, t) \in D \cap V$. Since $V \subseteq \Omega$, the hypotheses about $v$ imply $(\psi(t)-\varepsilon, t) \in\{v<0\} \cap V$. Thus for all small positive $\varepsilon, \psi(t)-\varepsilon<u(t)$, so $\psi(t) \leqslant u(t)$.] So $\psi-u$ has a maximum at 0 .

Now $v$ is strictly plurisubharmonic and $x-u(t)$ is a defining function for $\{v<0\}$ near 0 , so $u$ must be strictly superharmonic at 0 on complex lines through 0 in $U$. If $\zeta$ parametrizes such a line, then $u_{\zeta \zeta}(0)<0$. But $\psi-u$ has a maximum at 0 , so $(\psi-u)_{\zeta \zeta}(0) \leqslant 0$, whence $\psi_{\zeta \zeta}(0)<0$. Thus $\psi$ is also strictly superharmonic at 0 on complex lines through 0 in $U$, from which it follows 
that $\alpha$ is a strictly pseudoconvex boundary point of $\Omega$.

3. Existence of strictly pseudoconvex boundary points. We are now ready to prove a fairly general result about the existence of strictly pseudoconvex boundary points. This result has a number of direct consequences, including the characterization of the Shilov boundary of $A(D)$ mentioned in the Introduction.

THEOREM. Let $U_{1}, U$ be bounded open subsets of $\mathbf{R} \times \mathbf{C}^{n-1}$ with $\bar{U} \subseteq U_{1}$. Let $\psi \in C^{2}\left(U_{1}\right)$, let $\varepsilon_{1}>\varepsilon>0$, and let $D, V$ denote the following subsets of $\mathbf{C}^{n}$ :

$$
\begin{aligned}
& D=\left\{(x, t) \in \mathbf{R} \times U_{1} \mid \psi(t)-\varepsilon_{1}<x<\psi(t)\right\} ; \\
& V=\{(x, t) \in \mathbf{R} \times U \mid \psi(t)-\varepsilon<x<\psi(t)+\varepsilon\} .
\end{aligned}
$$

Suppose there is a plurisubharmonic function $u \in C_{\mathbf{R}}^{2}(D)$ for which $\sup _{D \backslash V} u<$ $\sup _{D} u$. Then $V$ contains a strictly pseudoconvex boundary point of $D$. (Note that $\partial D \cap V=\{(x, t) \in \mathbf{R} \times U \mid x=\psi(t)\}$ contains only $C^{2}$ boundary points of D.)

Proof. We may assume that $u$ is strictly plurisubharmonic on $D$, for $u$ may be replaced by $u+\delta \sum_{j=1}^{n}\left|z_{j}\right|^{2}$ if $\delta$ is a sufficiently small positive number. Let $K=\{(x, t) \in \mathbf{R} \times \bar{U} \mid x=\psi(t)\}$. For $0<s<\varepsilon_{1}$ define

$$
D_{s}=(s, 0)+D=\left\{(x, t) \in \mathbf{R} \times U_{1} \mid s+\psi(t)-\varepsilon_{1}<x<s+\psi(t)\right\},
$$

and note that each $D_{s}$ is a neighborhood of $K$ in $\mathbf{C}^{n}$. Define $u_{s}$ on $D_{s}$ by

$$
u_{s}(y, t)=u(y-s, t), \quad(y, t) \in D_{s},
$$

and let $m(s)=\max _{K} u_{s}$ for $0<s<\varepsilon_{1}$. Notice that $m$ is continuous on $\left(0, \varepsilon_{1}\right)$, that $\sup m=\sup u$, and that $m(s) \leqslant \sup _{D \backslash V} u$ when $\varepsilon \leqslant s<\varepsilon_{1}$. Choose $c$ with $\sup _{D \backslash V} u<c<\sup _{D} u$, and let

$$
r=\max \{x \in(0, \varepsilon) \mid m(s) \geqslant c\} .
$$

Then $m(r)=c$, and $u_{r}<c$ on $D_{r} \cap D$. Choose $\alpha \in K$ so that $u_{r}(\alpha)=c$. It is easily seen that $\alpha \in \partial D \cap V$. Observe that $u_{r}-c$ is strictly plurisubharmonic on $D_{r}$, that $\left(u_{r}-c\right)(\alpha)=0$, and that $u_{r}-c<0$ on $D_{r} \cap D$. By the above proposition, $\alpha$ is a strictly pseudoconvex boundary point of $D$; since $\alpha \in V$, this completes the proof of the Theorem.

REMARK. The assumption that $u$ is smooth in the Theorem and in Corollary 1 below is unnecessary. One need only approximate the given plurisubharmonic function by a smooth one defined on a slightly smaller domain in the usual way (as is done, e.g., for subharmonic functions in Theorem 1.6.11 in [5]), and observe that the boundary of the smaller domain may be taken to be a translate of the boundary of the original domain near the boundary points of interest.

COROllaRy 1. Let $D$ be an open subset of $\mathbf{C}^{n}$, and let $\alpha$ be a $C^{2}$ boundary point of $D$. If there is a "plurisubharmonic barrier" for $\alpha$ on $D$, then $\alpha$ is a limit 
of strictly pseudoconvex boundary points of D. (By a "plurisubharmonic barrier" we mean a function $u \in C_{\mathbf{R}}^{2}(D)$ such that $\lim _{\sup _{z \in D, z \rightarrow \alpha}} u(z)=0$ but for each neighborhood $V$ of $\alpha$ in $\mathbf{C}^{n}, \sup _{D \backslash V} u<0$.)

Proof. After a suitable change of coordinates the Theorem may be applied on a small neighborhood of $\alpha$.

Corollary 2. Suppose that $D$ is an open subset of $C^{n}$, that $\alpha$ is a $C^{2}$ boundary point of $D$, and that there is a function $f \in A(D)$ which peaks at $\alpha(f(\alpha)=1$ and $|f|<1$ on $\bar{D} \backslash\{\alpha\})$. Then $\alpha$ is a limit of strictly pseudoconvex boundary points.

Proof. Apply Corollary 1 in a neighborhood of $\alpha$ with $u=\log |f|$.

Corollary 3. Let $D$ be a bounded domain in $\mathbf{C}^{n}$ with $C^{2}$ boundary. Then the Shilov boundary of $A(D)$ is a subset of the closure of the strictly pseudoconvex boundary points of $A(D)$.

Proof. The Shilov boundary of a uniform algebra on a compact metric space is the closure of the set of peak points. Thus Corollary 3 follows from Corollary 2.

\section{REFERENCES}

1. H. J. Bremermann, On a generalized Dirichlet problem for plurisubharmonic functions and pseudo-convex domains. Characterization of Silov boundaries, Trans. Amer. Math. Soc. 91 (1959), 246-276. MR 25 \#227.

2. A. Debiard and B. Gauveau, Démonstration d'une conjecture de Bremermann sur la frontière de Silov d'un domaine faiblement pseudoconvexe, C. R. Acad. Sci. Paris 279 (1974), p. 407.

3. R. Epe, Charakterisierung des Schilowrandes von Holomorphiegebrieten, Schr. Math. Inst. Univ. Münster No. 25, (1963), 68 pp. MR $28 \# 2251$.

4. M. Hakim and N. Sibony, Frontière de Silov et spectre de $A(\bar{D})$ pour des domaines faiblement pseudoconvexe (preprint).

5. L. Hörmander, An introduction to complex analysis in several variables, Van Nostrand, Princeton, N.J., 1966. MR 34\# 2933.

6. J. J. Kohn, Global regularity for $\bar{\partial}$ on weakly pseudo-convex manifolds, Trans. Amer. Math. Soc. 181 (1973), 273-292. MR 49 \#9442.

7. P. Malliavin, Comportement à la frontière distinguée d'une fonction analytique de plusieurs variables, C. R. Acad. Sci. Paris 268 (1969), 380-381.

8. S_ Sur la répartition des zéros d'une fonction de la classe Nevanlinna dans le polydisque, C. R. Acad. Sci. Paris 271 (1970), 313-315.

9. Fonctions de Green d'un ouvert strictement pseudo-convexe et classe de Nevanlinna, C. R. Acad. Sci. Paris 278 (1974), 133-139.

10. P. Pflug, Über polynomiale Funktionen auf Holomorphiegebieten, Math. Z. 139 (1974), 133-139. MR 50 \#7579.

11. H. Rossi, Holomorphically convex sets in several complex variables, Ann. of Math. (2) 74 (1961), 470-493. MR 24 \# A3310.

12. E. M. Stein, Boundary behavior of holomorphic functions of several complex variables, Princeton Univ. Press, Princeton, N.J., 1972. 